NIST Special Publication 250-96

\title{
Dimensional measurement of nanostructures with scanning electron microscopy
}

K. A. Bertness

This publication is available free of charge from: https://doi.org/10.6028/NIST.SP.250-96

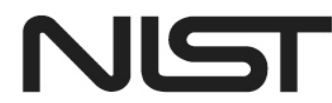

National Institute of Standards and Technology U.S. Department of Commerce 


\title{
NIST Special Publication 250-96
}

\section{Dimensional measurement of nanostructures with scanning electron microscopy}

\author{
K. A. Bertness \\ Applied Physics Division \\ Physical Measurement Laboratory
}

This publication is available free of charge from:

https://doi.org/10.6028/NIST.SP.250-96

September 2017

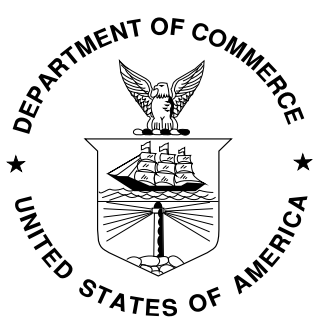

U.S. Department of Commerce Wilbur L. Ross, Jr., Secretary 
Certain commercial entities, equipment, or materials may be identified in this document in order to describe an experimental procedure or concept adequately. Such identification is not intended to imply recommendation or endorsement by the National Institute of Standards and Technology, nor is it intended to imply that the entities, materials, or equipment are necessarily the best available for the purpose.

National Institute of Standards and Technology Special Publication 250-96 Natl. Inst. Stand. Technol. Spec. Publ. 250-96, 22 pages (September 2017) CODEN: NSPUE2

This publication is available free of charge from: https://doi.org/10.6028/NIST.SP.250-96 


\begin{abstract}
Scanning electron microscopy (SEM) is widely used for the measurement of dimensions of nanostructures. This document describes the calibration of SEM magnification using the ASTM E766-14 practice with NIST Reference Material (RM) 8820 and the calculation of dimensional uncertainty in the use of the calibrated SEM to measure dimensions of a fabricated nanostructure. The dimensional measurements are performed as NIST Special
\end{abstract}

Test 15510 S.

\title{
Key words
}

Dimensional measurement; nanostructure; scanning electron microscopy.

\section{Table of Contents}

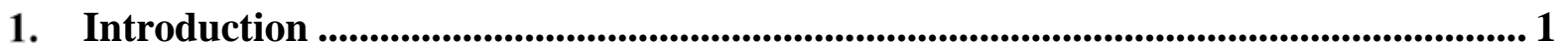

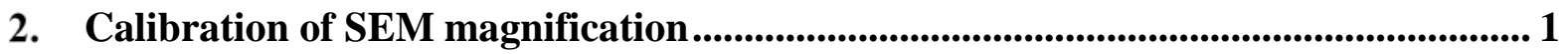

3. Application to specimens with unknown dimensions.................................................... 7

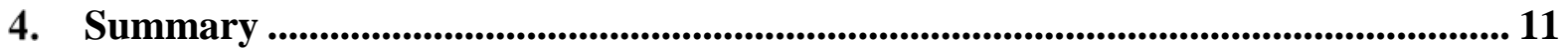

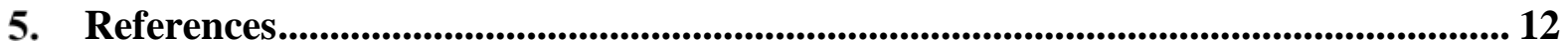

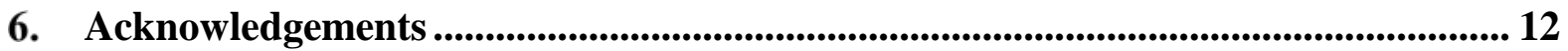

7. Appendix A. Example of an SEM Calibration Report (begins on the next page) ... 12

\section{List of Tables}

Table 1. Example of uncertainties for SEM magnification ...............................................6

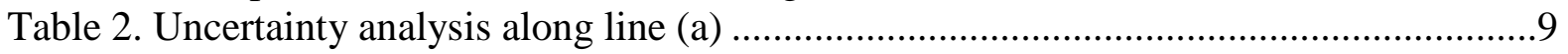

Table 3. Uncertainty analysis along line (b) ..................................................................10

Table 4. Examples of uncertainties for height of a nanostructure .....................................11

\section{List of Figures}

Fig. 1. Distribution of pixel measurements.....................................................................4

Fig. 2. Example of measurements on RM 8820 array 1C .................................................6

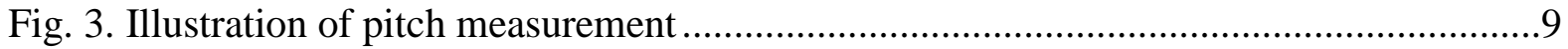

Fig. 4. Illustration of height measurement ................................................................... 11 


\section{Introduction}

Scanning electron microscopy (SEM) is widely used for the measurement of dimensions of nanostructures. This document describes the calibration of SEM magnification using the ASTM E766-14 [1] practice with NIST Reference Material (RM) 8820 [2] and the calculation of dimensional uncertainty in the use of the calibrated SEM to measure dimensions of a fabricated nanostructure. The dimensional measurements are performed as NIST Special Test 15510S.

\section{Calibration of SEM magnification}

As described by ASTM E766-14, the following calibration measurement conditions must be chosen to be the same as those used for test measurements: accelerating voltage, working distance, tilt angle, imaging mode (i.e., detector and spot size) and nominal magnification $M_{\text {nom. }}$ Each calibration is documented with a calibration report as described in Section 7 of ASTM E766-14. The calibration report includes a measurement of the temperature in the room to confirm that the temperature is within normal operating parameters for the SEM.

First, RM 8820 is imaged in the SEM. The artifact is placed so that the $x$ and $y$ directions of the images are parallel to the two axes of the calibration arrays for A-G type features (see Fig. 4 in Ref. 2) without any electronic rotation of the image. For clarity, we adopt the convention that the $x$ axis is the horizontal direction in Fig. 1 of Ref. 2 and the $y$ axis is the vertical direction. One of the features A-G is selected as appropriate for the particular magnification to be calibrated. Images are acquired of all four of the identical features with the same letter designation at the nominal magnification value $M_{\text {nom }}$ programmed into the SEM instrument. For example, if feature type C is chosen, calibration measurements will be taken for the $x$-direction on features $1 \mathrm{C}$ and $3 \mathrm{C}$, and calibration 
measurements will be taken for the $y$-direction on features $2 \mathrm{C}$ and $4 \mathrm{C}$, using the numerical row and column labels at the corners of the overall feature (see Fig. 4 in Ref. 2). The images are saved with the nominal length per pixel, $P_{\text {nom }}$.

To measure the height of the nanostructures, images will be taken with specimens at nonzero tilt angle $\theta$ in addition to measurements at $\theta=0$. To include the effects of mechanical tilt angle reproducibility in the uncertainty analysis, measurements at each angle setting are performed twice. In each of the two measurements, the tilt angle is set to a value $5^{\circ}$ greater than the target value, and then set to the target value, to minimize the effects of mechanical backlash. For $x$ and $y$ magnification calibration, $\theta$ is set to zero. A total of eight images are thus acquired for a magnification condition $\theta=0$, four each for $x$ and $y$. For nanostructure height measurements, $\theta$ is set to a nonzero value, with a typical value being $20^{\circ}$. The SEM used for this measurement service is designed such that increasing tilt rotates the specimen normal about the $x$ axis. For measurements with nonzero tilt, the $y_{\theta}$ values will be foreshortened by the tilt in the $y$ direction and nominally unchanged in the $x_{\theta}$ direction. A set of two images, one for each of the lettered features for $x_{\theta}$ and $y_{\theta}$, are recorded and then repeated after mechanically resetting the tilt angle, for a total of eight images taken at nonzero tilt angle.

Image analysis software is used on these images to determine a magnification correction factor $C$ that converts $M_{\text {nom }}$ to the actual calibrated magnification $M_{c a l}$, that is, $M_{c a l}$ $=C \cdot M_{n o m}$. The correction factor captures the magnification uncertainties in a number close to one over all the magnification and measurement conditions, and allows us to treat the nominal magnification numbers as constants with no associated uncertainty. There are several suitable image analysis programs available; the primary requirements are that these 
programs include an ability to enter the nominal conversion factor $P_{\text {nom }}$ from pixels to length independently for horizontal and vertical directions in the image and to conveniently measure the apparent length of features in the image after entering these conversion factors. The software program name and version number are recorded on the calibration report as a quality control practice. The value of $P_{\text {nom }}$ is provided by the SEM instrument based on $M_{\text {nom }}$, and the calibrated conversion factor $P_{\text {cal }}$ has the same relationship to $P_{\text {nom }}$ as $M_{\text {nom }}$ has to $M_{\text {cal }}$, that is, $P_{c a l}=C \cdot P_{\text {nom }}$. By measuring pitch features on RM 8820 with calibrated pitch $S_{\text {cal }}$ from Table 1 in Ref. 2, we can determine values for $C$ and its uncertainty.

One of the contributions to the uncertainty is the reproducibility of using the software to determine the number of pixels $n$ in a dimensional measurement. At a minimum, we expect this uncertainty to be half a pixel, and thus the minimum relative uncertainty $\sigma_{n} / n=0.5 / n$. To estimate the uncertainty due to software use, we measured a set of features five times each $(N=5)$. We measured a set of similar features ten times each $(N=10)$ to establish the effect of a greater number of samples. For each set, we calculated the average and standard deviation for the set, and these data are plotted in Fig. 1. The data show that the uncertainty due to pixel measurement is near to the ideal value, and fitting the data to a function of form $A$ / $n$, where $A$ is a constant, yields $A=0.78$ with $\sigma_{A}=0.06$. We thus conservatively estimate that $\sigma_{n} / n=0.84 / n$. For actual calibrations, the relative standard deviation of the calibration correction factor measurements may be larger than that predicted by the best fit equation. In this case, the pixel count uncertainty is adjusted so that the combined relative uncertainty in pixel count and angular reproducibility are at least equal to the measured relative standard deviation of the set of calibration correction factors. We assume that the pixel number uncertainties in the $x$ and $y$ direction are independent so that $\sigma_{n} / n=\sigma_{n, x} / n_{x}$ 
$=\sigma_{n, y} / n_{\mathrm{y}}$, for $n=n_{x}=n_{\mathrm{y}}$, and the data in Fig. 1 support this assumption. In principle, when features with different pixel numbers are combined for calibration averaging, each should be weighted by a different uncertainty. The data in Fig. 1 indicate that so long as features greater than approximately 250 pixels are chosen, a reasonable estimate for the relative uncertainty can be taken as $0.84 / 250=0.34 \%$.

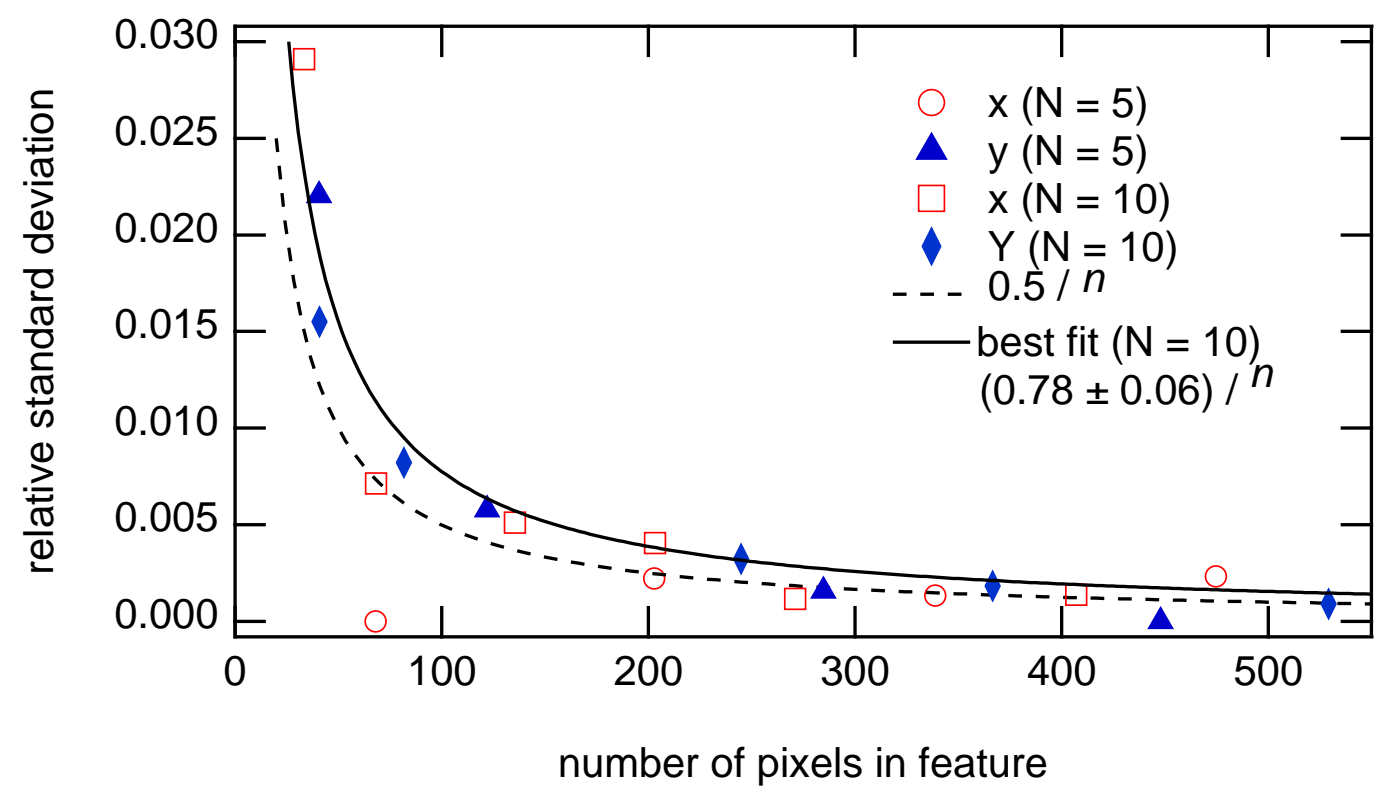

Fig. 1. Distribution of pixel measurements for repeated measurements of the same feature as a function of the total number of pixels in the measured feature.

As described above, for each magnification condition and each direction $\left(x, y, x_{\theta}\right.$, or $y_{\theta}$ ), there is a set of four images of nominally identical features, consisting of images of the two identical RM 8820 features (e.g., 1C and 3C) at two instances of setting the tilt angle. An example of an SEM calibration report is given in Appendix A. The apparent pitch $D$ of the lines in each image is measured five times using the image analysis program with $P_{\text {nom }}$ applied to the image, as illustrated in Fig. 2. A set of five values for $C$ can then be determined for each image using equation (1)

$$
C D=S_{\text {cal }} \cos (\theta) \text {, or } C=S_{\text {cal }} \cos (\theta) / D \text {. }
$$


Applying the rule that relative uncertainties of independent multiplicative factors add in quadrature [3] and using the fact that $D=n P_{\text {nom, }}$, we can write the uncertainty propagation for $C$ as

$$
\left(\sigma_{C} / C\right)^{2}=\left(\sigma_{s} / S_{\text {cal }}\right)^{2}+\left(\sigma_{n} / n\right)^{2}+\tan ^{2}(\theta) \sigma_{\theta}{ }^{2},
$$

where we have used the partial derivative of $\cos (\theta)$ with respect to $\theta$ to separate the tilt angle uncertainty from the functional dependence in Eqn. 1. The first term in Eqn. 2 comes from Table 1 in Ref. 2, and the second term has been shown above to be $0.34 \%$ or less for features with pixel size of 250 or more. To estimate the effect of tilt angle $\theta$ on the calibration uncertainty, we first note that the factor of $\tan \theta$ indicates that the contribution of the angle uncertainty to the total dimensional uncertainty becomes vanishingly small as $\theta$ approaches 0. Secondly, we note that for nonzero $\theta$, we could estimate the last term in Eqn. 2 by taking the relative standard deviation of the $C$ values we calculate from calibration data and subtracting the first two terms in Eqn. 2 from that value. However, we find that the relative uncertainty in $S_{c a l}$ is approximately an order of magnitude larger than the relative standard deviation for a typical calibration data set because $S_{\text {cal }}$ includes systematic uncertainty and variation within the ensemble of reference materials created. The angle uncertainty is thus shown to have a small effect on the total calibration uncertainty, even at $\theta=20^{\circ}$. As a second estimate of the angle-related uncertainty, we compare the ten values of $C$ for one setting of angle $\theta$ to those of the ten values of the other mechanical setting to the same value of $\theta$ for a calibration data set with nonzero $\theta$. As detailed in Appendix A, the mean and standard deviation of $C$ for set 1 are 1.0148 and 0.0052 , respectively, and for set 2, 1.0125 and 0.0030 . The average values agree to within their standard deviation $(k=1)$, which confirms that the effect of angular uncertainty is small. We can estimate the value of $\tan (\theta)$ 
$\sigma_{\theta}$ as at most the difference between the two means for the two sets normalized to the mean, 0.0025. An example of an uncertainty calculation for $C$ is given in Table 1 .

\begin{tabular}{|l|c|}
\hline Source & $\begin{array}{c}\text { Standard relative uncertainty (type) } \\
(\%)\end{array}$ \\
\hline $\begin{array}{l}\text { RM8820 pitch, } 2000 \mathrm{~nm} \text { nominal } \\
\text { (feature } \mathrm{G}): \sigma_{S} / S_{\text {cal }}\end{array}$ & $3.23(\mathrm{~B})$ \\
\hline Pixel count: $\sigma_{n} / n$ & $0.34(\mathrm{~A})$ \\
\hline Tilt angle: $\tan (\theta) \sigma_{\theta}$ & $0.25(\mathrm{~A})$ \\
\hline Combined relative uncertainty & 3.26 \\
\hline Expanded relative uncertainty $(k=2)$ & 6.51 \\
\hline
\end{tabular}

Table 1. Example of uncertainties for SEM magnification correction factor $C$ for the $y$ direction with nominal magnification 10,000 and tilt angle of $20^{\circ}$.

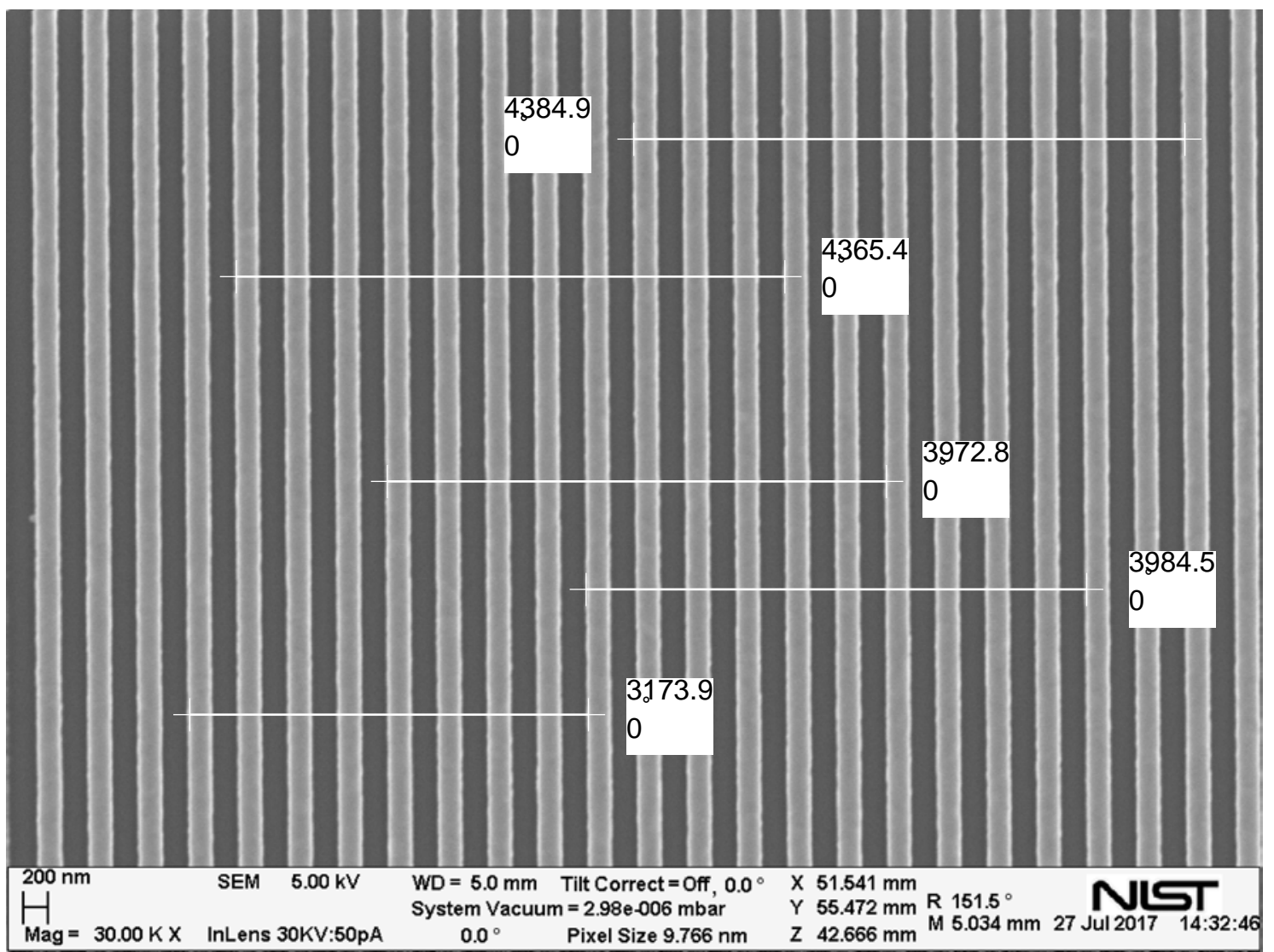

Figure 2. Example of measurements on RM 8820 array 1C, with nominal pitch $400 \mathrm{~nm}$ and measured pitch $402 \pm 20 \mathrm{~nm}, k=2$. 


\section{Application to specimens with unknown dimensions}

The nanostructures specimens to be measured are mounted in the SEM and imaged with operating conditions identical to those used in the magnification calibration process, as detailed in the previous section. These images are analyzed with the same image analysis program used to analyze the SEM calibration images, but instead of using $P_{\text {nom }}$ in the analysis program, we use the calibrated value $P_{c a l}=C \cdot P_{\text {nom }}$ for measurements with zero tilt angle.

The calibrated feature length $L_{c a l}$ is then the length determined with the image analysis program after applying the calibrated pixel conversion factors. The measurements are repeated ten times for each feature, and the mean value is used as $L_{c a l}$. For greatest generality, in the calculation of the uncertainty, we consider the case in which the feature is oriented at an angle $\varphi$ relative to the $x$ axis. The image program calculates the feature length as

$$
L_{c a l}=\left[\left(P_{c a l, x} \cdot n_{x}\right)^{2}+\left(P_{c a l, y} \cdot n_{y}\right)^{2}\right]^{1 / 2},
$$

where $n_{x}$ and $n_{y}$ are the number of pixels in the $x$ and $y$ direction, respectively, for a rectangle that just encloses the feature. The uncertainty in the length $\sigma_{L}$ is calculated by separately considering the uncertainty in the projection of the length along each axis, assuming input quantities are independent:

$$
\sigma_{L x} /\left(P_{c a l, x} \cdot n_{x}\right)=\left[\left(\sigma_{n, x} / n_{x}\right)^{2}+\left(\sigma_{P x} / P_{c a l, x}\right)^{2}\right]^{1 / 2}
$$

The first term in the square brackets is the pixel number uncertainty quantified in Fig. 1. Although it is generally possible to carry out the calibration pitch measurements with dimensions that are at least 250 pixels, nanostructures under test may not meet that criterion and the actual value of $0.78 / n$ is used to estimate the pixel number uncertainty for $n<250$. The second term is equal to the relative uncertainty in the magnification correction factor for 
the $x$ direction. A similar equation can be written for $\sigma_{L y}$, and the combined uncertainty in $L_{c a l}$ becomes (again assuming $n_{x}$ and $n_{y}$ are independent):

$$
\begin{aligned}
\sigma_{L}{ }^{2}= & \left(L_{x} / L_{c a l}\right)^{2} \sigma_{L x}{ }^{2}+\left(L_{y} / L_{c a l}\right)^{2} \sigma_{L y}{ }^{2} \\
= & \cos ^{2}(\varphi)\left[\left(P_{\text {nom }} C_{x} \sigma_{\mathrm{n}}\right)^{2}+\left(P_{\text {nom }} n_{x} \sigma_{C x}\right)^{2}\right]+\sin ^{2}(\varphi)\left[\left(P_{\text {nom }} C_{y} \sigma_{\mathrm{n}}\right)^{2}+\left(P_{\text {nom }} n_{y} \sigma_{C y}\right)^{2}\right] \\
= & \cos ^{2}(\varphi)\left[\left(L_{\text {cal }} \cos (\varphi)\right)^{2}\left(\sigma_{n, x} / n_{x}\right)^{2}+\left(L_{\text {cal }} \cos (\varphi)\right)^{2}\left(\sigma_{C x} / C_{x}\right)^{2}\right] \\
& \quad+\sin ^{2}(\varphi)\left[\left(L_{c a l} \sin (\varphi)\right)^{2}\left(\sigma_{n, y} / n_{x}\right)^{2}+\left(L_{c a l} \sin (\varphi)\right)^{2}\left(\sigma_{C y} / C_{y}\right)^{2}\right] \\
& \quad L_{c a l}{ }^{2}\left\{\cos ^{4}(\varphi)\left[\left(\sigma_{n, x} / n_{x}\right)^{2}+\left(\sigma_{C x} / C_{x}\right)^{2}\right]+\sin ^{4}(\varphi)\left[\left(\sigma_{n, y} / n_{y}\right)^{2}+\left(\sigma_{C y} / C_{y}\right)^{2}\right]\right\}
\end{aligned}
$$

where we have made use of the relationships $L_{x}=L_{c a l} \cos (\varphi)=P_{c a l, x} \cdot n_{x}=P_{n o m} \cdot C_{x} \cdot n_{x}$ and $L_{y}=L_{c a l} \sin (\varphi)=P_{c a l, y} \cdot n_{y}=P_{n o m} \cdot C_{y} \cdot n_{y}$ and we have added subscripts to the calibration correction factors to make explicit that these factors and their uncertainties vary from dimension to dimension. The terms related to pixel count can be grouped together to calculate a weighted combined pixel count relative uncertainty defined as $\cos ^{4}(\varphi)\left(\sigma_{n, x} / n_{x}\right)^{2}+$ $\sin ^{4}(\varphi)\left(\sigma_{n, y} / n_{y}\right)^{2}$

Examples of lateral dimension measurements are given in Fig. 3 with the uncertainty calculations given in Tables 2 and 3. For line (a) in Fig. 3, the number of pixels in the pitch feature is 506 so the standard relative uncertainty in pixel count of $0.84 / 506$ is applied. The contribution of the small angle $\varphi \sim 0.2^{\circ}$ does not contribute to the overall uncertainty because $\sin ^{4}(\varphi)=1.5 \times 10^{-10}$, so we consider only uncertainties in the $x$ direction. The mean of ten measurements of line (a) is $S=5003 \mathrm{~nm}$ with a relative standard deviation of $0.13 \%$, less than our estimated pixel count relative uncertainty, so we use the larger, estimated value. The expanded uncertainty in $S$ is $(5003 \mathrm{~nm})(0.050)=250 \mathrm{~nm}$. We report the pitch as 5000 $\mathrm{nm} \pm 250 \mathrm{~nm}(k=2)$. For line (b), we estimate the $x$ and $y$ pixel count relative uncertainties from $0.84 / n$, and combine them as described above with angular weighting factors 
$\cos ^{4}\left(60.2^{\circ}\right)=0.061$ and $\sin ^{4}\left(60.2^{\circ}\right)=0.567$ to yield an estimated pixel count relative uncertainty of $0.16 \%$. The mean pitch line (b) is measured to be $S=4984 \mathrm{~nm}$ with a relative standard deviation of $0.23 \%$. The latter uncertainty is larger than the estimated value for pixel count relative uncertainty, so we use the larger, actual value. The expanded uncertainty in $S$ is $(4984 \mathrm{~nm})(0.040)=200 \mathrm{~nm}$. We report the pitch as $5000 \mathrm{~nm} \pm 200 \mathrm{~nm}(k=2)$.

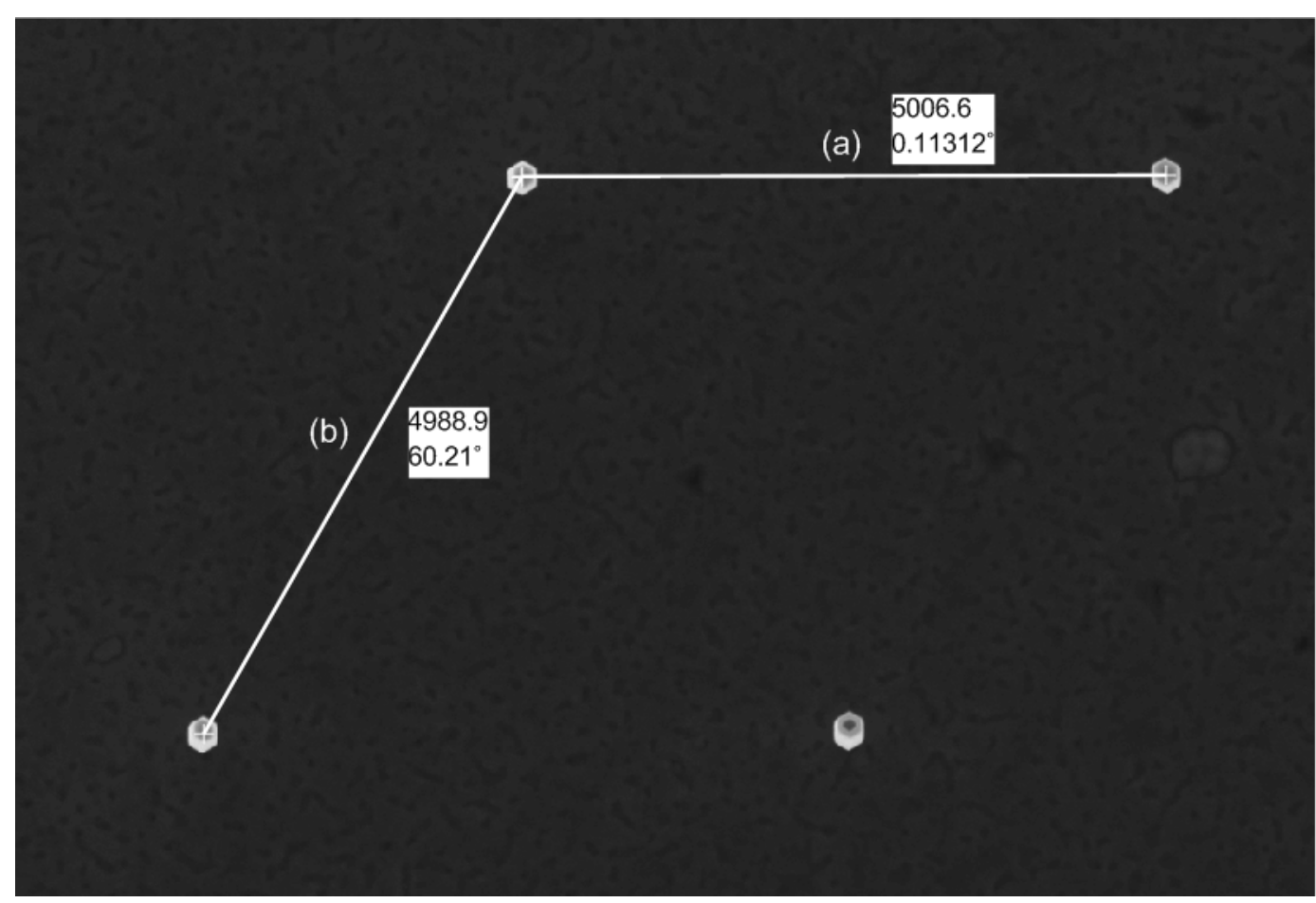

Fig. 3. Illustration of pitch measurement for tilt angle $0^{\circ}$ using $P_{c a l}$ of $9.875 \mathrm{~nm} / \mathrm{pixel}$ and $9.885 \mathrm{~nm} /$ pixel, respectively, for $x$ and $y$, as derived in the SEM calibration example in Appendix A for nominal magnification of 30,000. Only one of the ten measurements is illustrated for each line. See Tables 2 and 3 for uncertainty analysis of lines (a) and (b), respectively.

\begin{tabular}{|l|c|}
\hline Source & $\begin{array}{c}\text { Standard relative uncertainty (type) } \\
(\%)\end{array}$ \\
\hline Correction factor: $\sigma_{C x} / C_{x}$ & $2.5(\mathrm{~B})$ \\
\hline Pixel count: $\sigma_{n, x} / n_{x}$ & $0.17(\mathrm{~A})$ \\
\hline Combined relative uncertainty & 2.51 \\
\hline $\begin{array}{l}\text { Expanded relative uncertainty } \\
(k=2)\end{array}$ & 5.0 \\
\hline
\end{tabular}

Table 2. Uncertainty analysis for the pitch of two nanostructures along line (a) in Fig. 3. 


\begin{tabular}{|l|c|}
\hline Source & $\begin{array}{c}\text { Standard relative uncertainty (type) } \\
(\%)\end{array}$ \\
\hline Correction factor: $\sigma_{C x} / C_{x}$ & $2.5(\mathrm{~B})$ \\
\hline Correction factor: $\sigma_{C y} / C_{y}$ & $2.5(\mathrm{~B})$ \\
\hline Pixel count: $\sigma_{n, x} / n_{x}, n_{x} \approx 254$ & $0.33(\mathrm{~A})$ \\
\hline Pixel count: $\sigma_{n, y} / n_{y}, n_{y} \approx 441$ & $0.19(\mathrm{~A})$ \\
\hline $\begin{array}{l}\text { Combined pixel count relative } \\
\text { uncertainty (see text) }\end{array}$ & 0.16 \\
\hline Combined relative uncertainty & 2.0 \\
\hline Expanded relative uncertainty $(k=2)$ & 4.0 \\
\hline
\end{tabular}

Table 3. Uncertainty analysis for the pitch of two nanostructures along line (b) in Fig. 3.

For measurements of the height of nanostructures made at nonzero tilt angle, we constrain the sample orientation so that the dimension to be measured is parallel to the $y$ axis $\left(\varphi=90^{\circ}\right)$ and the tilt angle $\theta$ is applied about the $x$ axis. The height $h$ of the nanostructure is then given by the apparent dimension $d$ in the image divided by $\sin (\theta)$, i. e., $h=d / \sin (\theta)$. Because $d$ itself is equal to the product of $P_{c a l, y} \cdot n_{y}=C_{y \theta} \cdot P_{n o m} \cdot n_{y}$, we can write the uncertainty for $h$ as

$$
\left(\sigma_{h} / h\right)^{2}=\left(\sigma_{n, y} / n_{y}\right)^{2}+\left(\sigma_{C y} / C_{y \theta}\right)^{2}+\cot ^{2}(\theta) \sigma_{\theta}^{2} .
$$

We have included $\theta$ in the subscript of $C_{y \theta}$ as a reminder that this is the calibration correction factor for a nonzero value of $\theta$, and we again assume the variations in the input variables are independent. The first two terms on the right have been evaluated in the previous section. The third term can be related to our estimate for $\tan (\theta) \sigma_{\theta}=0.0025$ by rewriting $\cot (\theta) \sigma_{\theta}$ as $\cot ^{2}(\theta) \tan (\theta) \sigma_{\theta},=(7.55)(0.0025)=0.0189$ for $\theta=20^{\circ}$. An example of a height measurement is given in Fig. 4 with the uncertainty calculation given in Table 4 . The mean height of the object $h$ is measured to be $(2192 \mathrm{~nm}) / \sin \left(20^{\circ}\right)=6409 \mathrm{~nm}$ with a relative standard deviation of $0.57 \%$, but we use the larger estimated value of $0.78 / 74=$ $1.05 \%$ for the pixel count relative uncertainty. The expanded uncertainty in $h$ is (6409 $\mathrm{nm})(0.078)=500 \mathrm{~nm}$. We report the height as $6400 \mathrm{~nm} \pm 500 \mathrm{~nm}(k=2)$. 


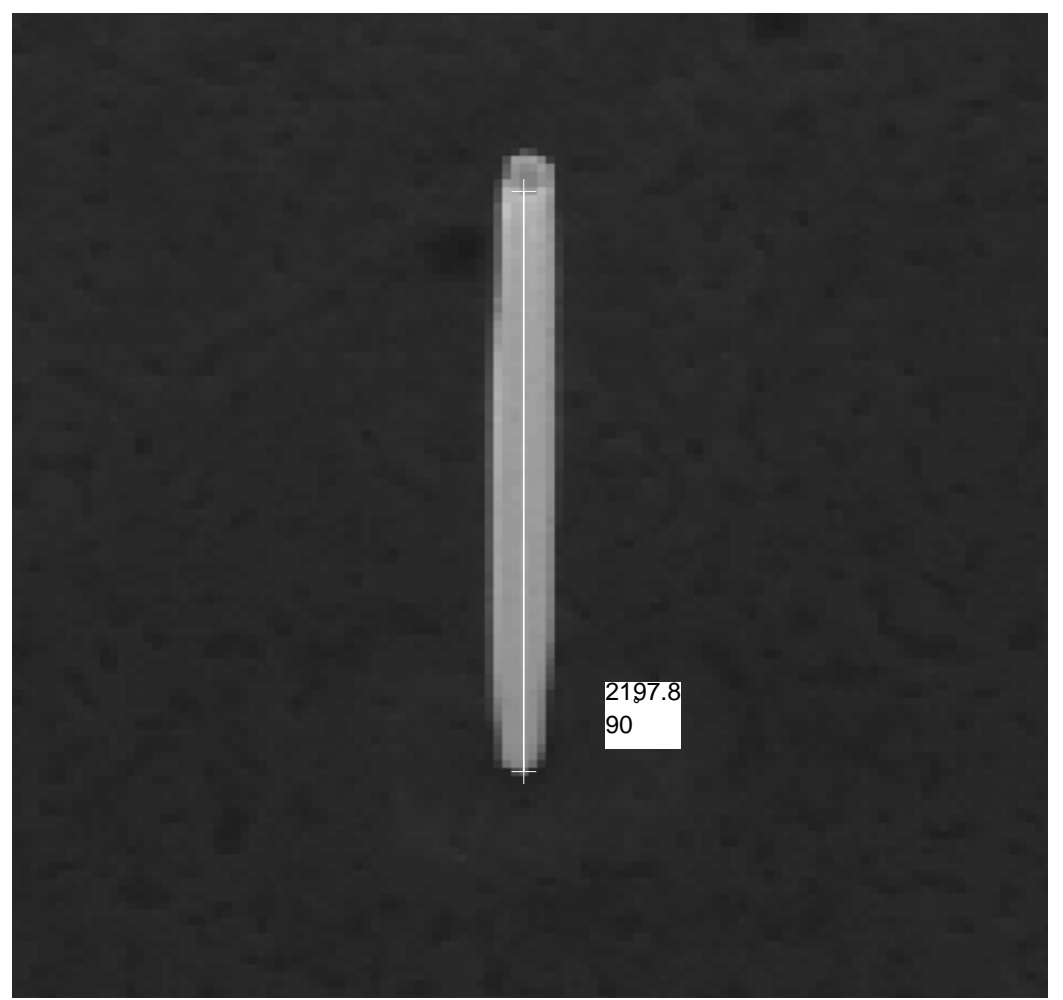

Fig. 4. Illustration of height measurement for tilt angle $20^{\circ}$ using $P_{\text {cal }}$ of $29.6 \mathrm{~nm} / \mathrm{pixel}$ and $29.7 \mathrm{~nm} /$ pixel, respectively, for $x$ and $y$, as derived in the SEM calibration example in Appendix A for nominal magnification of 10,000.

\begin{tabular}{|l|c|}
\hline Source & $\begin{array}{c}\text { Standard relative uncertainty (type) } \\
(\%)\end{array}$ \\
\hline Correction factor: $\sigma_{C y \theta} / C_{y \theta}$ & $3.24(\mathrm{~B})$ \\
\hline Pixel count: $\sigma_{n, y} / n_{y}$ & $1.05(\mathrm{~A})$ \\
\hline Tilt angle: $\cot (\theta) \sigma_{\theta}$ & $1.89(\mathrm{~A})$ \\
\hline Combined relative uncertainty & 3.9 \\
\hline $\begin{array}{l}\text { Expanded relative uncertainty } \\
(k=2)\end{array}$ & 7.8 \\
\hline
\end{tabular}

Table 4. Example of uncertainties for height of a nanostructure taken at a tilt angle $\theta=20^{\circ}$ based on the example calibration in Appendix A and the number of pixels for the apparent dimension in Fig. 4.

\section{Summary}

We have illustrated methods for calibrated measurements of lateral dimensions of nanostructures at zero tilt angle and nanostructure height at finite tilt angle. We also show uncertainty analysis and traceability methods making use of the NIST RM 8820 calibration 
artifact. For typical lateral measurements, the systematic error in the feature size of the calibration artifact is the major source of uncertainty. For height measurements, the uncertainty in tilt angle can become significant. Pixel count uncertainty can be minimized by adjusting magnification so that the features of interest extends for at least 250 pixels. Expanded uncertainties $(k=2)$ are on the order of $5 \%$ to $8 \%$ with these methods.

\section{References}

[1] ASTM E766-14, "Standard Practice for Calibrating the Magnification of a Scanning Electron Microscope,” editorially corrected in May 2016.

[2] Report of Investigation issued by NIST for Reference Material 8820, Scanning Electron Microscope Calibration Artifact, available as a free download at https://www-s.nist.gov/srmors/view_cert.cfm?srm=8820

[3] "Evaluation of measurement data — Guide to the expression of uncertainty in measurement,” Joint Committee for Guides in Metrology (JCGM) 100: 2008, pg. 20. Available as a free download at http://www.bipm.org/en/publications/guides/

\section{Acknowledgements}

We thank Jolene Splett for providing statistical review of the uncertainty analysis in this document, and Paul Blanchard for critical reading of the manuscript.

Appendix A. Example of an SEM Calibration Report (begins on the next page) 


\section{Scanning Electron Microscope (SEM) Calibration Report NIST, 325 Broadway, Boulder, CO 80305}

Instrument Name* and Location: Zeiss Auriga, Bldg 81, Room 1C109

Calibration Method Compliant with ASTM E766-14

Calibration artifact used: NIST RM 8820

Calibration pattern(s) used:

Feature G: $2 \mu \mathrm{m}$ pitch nominal, Measured $2.010 \mu \mathrm{m} \pm 0.13 \mu \mathrm{m}(k=2)$

Feature C: $400 \mathrm{~nm}$ pitch nominal, Measured $0.402 \mu \mathrm{m} \pm 0.02 \mu \mathrm{m}(k=2)$

Calibration Corrections Summary (relative to default SEM calibrations)

\begin{tabular}{|l|l|l|l|l|}
\hline Magnification /Tilt & X correction & Rel. unc. ( $\mathbf{k = 2})$ & $\begin{array}{l}\mathbf{Y} \\
\text { correction }\end{array}$ & Rel. unc. ( $\boldsymbol{k}=2$ ) \\
\hline $30,000 / 0^{\circ}$ & 1.0112 & $5.0 \%$ & 1.0122 & $5.0 \%$ \\
\hline $10,000 / 20^{\circ}$ & 1.0118 & $6.5 \%$ & 1.0137 & $6.5 \%$ \\
\hline
\end{tabular}

SEM Operating Conditions:

Accelerating voltage: $5 \mathrm{kV}$

Aperture Size: $30 \mu \mathrm{m}$

Working Distance: $5.0 \mathrm{~mm}$

Detector: In Lens

Measurement date: 27 July 2017 Measurement time: 1:30 pm

Room temperature: $22{ }^{\circ} \mathrm{C}$

Software Analysis Program and Version*: Igor 7.0.4.1

*Software program and SEM are identified only to adequately specify this procedure. In no case does such identification imply recommendation or endorsement by the National Institute of Standards and Technology, nor does it imply that these products are necessarily the best available for the purpose.

Other comments: none

Report Prepared By: Kris A. Bertness

Signature:

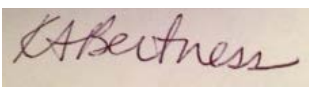

Date: 7 August 2017 
Calibration Data

Image names: RM_1C_Img_001, RM_1C_Img_002, RM_3C_Img_001, RM_3C_Img_002 Dimension: $X$ Magnification: 30,000 Tilt: $0^{\circ}$

\begin{tabular}{|l|l|l|}
\hline Actual $(\mathrm{nm})$ & $\begin{array}{l}\text { Measured } \\
(\mathrm{nm})\end{array}$ & Correction \\
\hline 4020 & 3972.8 & 1.0119 \\
\hline 4422 & 4365.4 & 1.0130 \\
\hline 4020 & 3984.5 & 1.0089 \\
\hline 3216 & 3173.9 & 1.0133 \\
\hline 4422 & 4384.9 & 1.0085 \\
\hline 3618 & 3584.1 & 1.0095 \\
\hline 2412 & 2382.9 & 1.0122 \\
\hline 5628 & 5576.4 & 1.0093 \\
\hline 4422 & 4365.4 & 1.0130 \\
\hline 2814 & 2783.3 & 1.0110 \\
\hline 4020 & 3974.8 & 1.0114 \\
\hline 3216 & 3173.9 & 1.0133 \\
\hline 5226 & 5166.2 & 1.0116 \\
\hline 2814 & 2783.3 & 1.0110 \\
\hline 4020 & 3974.8 & 1.0114 \\
\hline 4422 & 4384.9 & 1.0085 \\
\hline 3618 & 3574.4 & 1.0122 \\
\hline 5628 & 5576.4 & 1.0093 \\
\hline 3216 & 3164.2 & 1.0164 \\
\hline 4422 & 4384.9 & 1.0085 \\
\hline & & \\
\hline
\end{tabular}

Average of Correction values: $1.0112 \quad$ Relative standard deviation $(k=1): 0.21 \%$ Standard Relative Uncertainty in Correction Value (see table below): $2.50 \%$

\begin{tabular}{|l|c|}
\hline Source & $\begin{array}{c}\text { Standard relative uncertainty (type) } \\
(\%)\end{array}$ \\
\hline $\begin{array}{l}\text { RM8820 pitch, } 400 \mathrm{~nm} \text { nominal } \\
\text { (feature C) }\end{array}$ & $2.49(\mathrm{~B})$ \\
\hline Pixel count uncertainty & $0.34(\mathrm{~A})$ \\
\hline Angular reproducibility & $0(\mathrm{~A})$ \\
\hline Combined relative uncertainty & 2.51 \\
\hline $\begin{array}{l}\text { Expanded relative uncertainty } \\
(k=2)\end{array}$ & 5.0 \\
\hline
\end{tabular}


Calibration Data

Image names: RM_2C_Img_001, RM_2C_Img_002, RM_4C_Img_001, RM_4C_Img_002 Dimension: $Y$ Magnification: 30,000 Tilt: $0^{\circ}$

\begin{tabular}{|l|l|l|}
\hline Actual (nm) & $\begin{array}{l}\text { Measured } \\
(\mathrm{nm})\end{array}$ & Correction \\
\hline 2814 & 2783.3 & 1.0110 \\
\hline 4422 & 4375.2 & 1.0107 \\
\hline 3216 & 3173.9 & 1.0133 \\
\hline 3618 & 3584.1 & 1.0095 \\
\hline 3618 & 3574.4 & 1.0122 \\
\hline 3618 & 3564.6 & 1.0150 \\
\hline 4020 & 3974.8 & 1.0114 \\
\hline 2412 & 2382.9 & 1.0122 \\
\hline 4824 & 4756 & 1.0143 \\
\hline 3216 & 3144.7 & 1.0227 \\
\hline 3216 & 3173.9 & 1.0133 \\
\hline 4824 & 4765.8 & 1.0122 \\
\hline 3216 & 3173.9 & 1.0133 \\
\hline 3618 & 3574.4 & 1.0122 \\
\hline 3618 & 3574.4 & 1.0122 \\
\hline 3216 & 3183.7 & 1.0102 \\
\hline 2412 & 2392.7 & 1.0081 \\
\hline 4422 & 4365.4 & 1.0130 \\
\hline 2814 & 2783.3 & 1.0110 \\
\hline 3216 & 3193.5 & 1.0071 \\
\hline & & \\
\hline
\end{tabular}

Average of Correction values: $1.0122 \quad$ Relative standard deviation $(k=1): 0.31 \%$ Standard Relative Uncertainty in Correction Value (see table below): $2.51 \%$

\begin{tabular}{|l|c|}
\hline Source & $\begin{array}{c}\text { Standard relative uncertainty (type) } \\
(\%)\end{array}$ \\
\hline $\begin{array}{l}\text { RM8820 pitch, } 400 \mathrm{~nm} \text { nominal } \\
\text { (feature C) }\end{array}$ & $2.49(\mathrm{~B})$ \\
\hline Pixel count uncertainty & $0.34(\mathrm{~A})$ \\
\hline Angular reproducibility & $0(\mathrm{~A})$ \\
\hline Combined relative uncertainty & 2.51 \\
\hline $\begin{array}{l}\text { Expanded relative uncertainty } \\
(k=2)\end{array}$ & 5.0 \\
\hline
\end{tabular}


Calibration Data

Image names: RM_1Gt20_Img_001， RM_1Gt20_Img_002， RM_3Gt20_Img_001, RM_3Gt20_Img_002

Dimension: $X_{\theta}$ Magnification: 10,000 Tilt: $20^{\circ}$

\begin{tabular}{|l|l|l|}
\hline Actual (nm) & $\begin{array}{l}\text { Measured } \\
(\mathrm{nm})\end{array}$ & Correction \\
\hline 10050 & 9932.7 & 1.0118 \\
\hline 10050 & 9903.4 & 1.0148 \\
\hline 8040 & 7969.6 & 1.0088 \\
\hline 14070 & 13947 & 1.0088 \\
\hline 10050 & 9932.7 & 1.0118 \\
\hline 12060 & 11954 & 1.0089 \\
\hline 12060 & 11925 & 1.0113 \\
\hline 10050 & 9903.4 & 1.0148 \\
\hline 12060 & 11925 & 1.0113 \\
\hline 14070 & 13917 & 1.0110 \\
\hline 12060 & 11896 & 1.0138 \\
\hline 8040 & 7940.3 & 1.0126 \\
\hline 10050 & 9932.7 & 1.0118 \\
\hline 14070 & 13888 & 1.0131 \\
\hline 12060 & 11896 & 1.0138 \\
\hline 12060 & 11896 & 1.0138 \\
\hline 10050 & 9932.7 & 1.0118 \\
\hline 14070 & 13859 & 1.0152 \\
\hline 14070 & 13947 & 1.0088 \\
\hline 10050 & 9962 & 1.0088 \\
\hline & & \\
\hline
\end{tabular}

Average of Correction values: $1.0118 \quad$ Relative standard deviation $(k=1): 0.21 \%$ Standard Relative Uncertainty in Correction Value (see table below): $2.50 \%$

\begin{tabular}{|l|c|}
\hline Source & $\begin{array}{c}\text { Standard relative uncertainty (type) } \\
(\%)\end{array}$ \\
\hline $\begin{array}{l}\text { RM8820 pitch, } 2 \mu \mathrm{m} \text { nominal } \\
\text { (feature G) }\end{array}$ & $3.23(\mathrm{~B})$ \\
\hline Pixel count uncertainty & $0.34(\mathrm{~A})$ \\
\hline Angular reproducibility & $0(\mathrm{~A})$ \\
\hline Combined relative uncertainty & 3.25 \\
\hline $\begin{array}{l}\text { Expanded relative uncertainty } \\
(k=2)\end{array}$ & 6.5 \\
\hline
\end{tabular}


Calibration Data

Image names: RM_2Gt20_Img_001， RM_2Gt20_Img_002， RM_4Gt20_Img_001, RM_4Gt20_Img_002

Dimension: $Y_{\theta}$ Magnification: 10,000 Tilt: $20^{\circ}$

\begin{tabular}{|l|l|l|}
\hline Actual $(\mathrm{nm})$ & Measured $(\mathrm{nm})$ & Correction \\
\hline 9443.45 & 9317.4 & 1.0135 \\
\hline 11332.14 & 11251 & 1.0072 \\
\hline 7554.76 & 7412.9 & 1.0191 \\
\hline 7554.76 & 7471.5 & 1.0111 \\
\hline 15109.52 & 14914 & 1.0131 \\
\hline 9443.45 & 9346.7 & 1.0104 \\
\hline 13220.83 & 13068 & 1.0117 \\
\hline 9443.45 & 9288.1 & 1.0167 \\
\hline 9443.45 & 9376 & 1.0072 \\
\hline 11332.14 & 11222 & 1.0098 \\
\hline 13220.83 & 13068 & 1.0117 \\
\hline 7554.76 & 7412.9 & 1.0191 \\
\hline 9443.45 & 9346.7 & 1.0104 \\
\hline 9443.45 & 9229.5 & 1.0232 \\
\hline 9443.45 & 9258.8 & 1.0199 \\
\hline 11332.14 & 11163 & 1.0152 \\
\hline 11332.14 & 11163 & 1.0152 \\
\hline 7554.76 & 7471.5 & 1.0111 \\
\hline 7554.76 & 7442.2 & 1.0151 \\
\hline 11332.14 & 11193 & 1.0124 \\
\hline & &
\end{tabular}

Average of Correction values: $1.0137 \quad$ Relative standard deviation $(k=1): 0.42 \%$ Standard Relative Uncertainty in Correction Value (see table below): $3.26 \%$

\begin{tabular}{|l|c|}
\hline Source & $\begin{array}{c}\text { Standard relative uncertainty (type) } \\
(\%)\end{array}$ \\
\hline $\begin{array}{l}\text { RM8820 pitch, } 2 \mu \mathrm{m} \text { nominal } \\
\text { (feature G) }\end{array}$ & $3.23(\mathrm{~B})$ \\
\hline Pixel count uncertainty & $0.34(\mathrm{~A})$ \\
\hline Angular reproducibility & $0.25(\mathrm{~A})$ \\
\hline Combined relative uncertainty & 3.26 \\
\hline $\begin{array}{l}\text { Expanded relative uncertainty } \\
(k=2)\end{array}$ & 6.5 \\
\hline
\end{tabular}


Separation of $\mathbf{Y}_{\theta}$ Correction Factor Data into Sets for Each Setting of Tilt Angle

\begin{tabular}{|l|l|}
\hline Set 1: & Set 2: \\
RM_2Gt20_Img_001, & RM_2Gt20_Img_002, \\
RM_4Gt20_Img_001 & RM_4Gt20_Img_002 \\
\hline 1.0135 & 1.0104 \\
\hline 1.0072 & 1.0117 \\
\hline 1.0191 & 1.0167 \\
\hline 1.0111 & 1.0072 \\
\hline 1.0131 & 1.0098 \\
\hline 1.0117 & 1.0152 \\
\hline 1.0191 & 1.0152 \\
\hline 1.0104 & 1.0111 \\
\hline 1.0232 & 1.0151 \\
\hline 1.0199 & 1.0124 \\
\hline Mean: 1.0148 & Mean: 1.0125 \\
\hline Std. Dev.: 0.0052 & Std. Dev.: 0.0030 \\
\hline Rel. Std. Dev.: $0.51 \%$ & Rel. Std. Dev.: $0.30 \%$ \\
\hline
\end{tabular}

